\title{
Treatment approach in patients with oral squamous - cell carcinoma
}

\author{
Mihaela Furnadzhieva ${ }^{1}$, Svetoslav Slavkov ${ }^{2}$, Daniel \\ Markov $^{2}$, Rossen Kolarov ${ }^{2}$, Assya Krasteva'.
}

1. Faculty of dental medicine, Department of oral and imaging diagnostic, Medical University, Sofia, Bulgaria;

2. University Specialized Hospital for Maxillofacial Surgery, Medical University - Sofia, Bulgaria;

\begin{abstract}
Oral cancer is currently the sixth most common carcinoma worldwide and squamous-cell carcinoma accounts for more than $90 \%$ of all cases. The earliest diagnosis of squamous-cell carcinoma, the better the outcome of the treatment and the prognosis. The choice of treatment depends on tumour stage. Most early squamous-cell carcinoma cases are amenable to surgical curative treatment while advanced cases commonly receive combined palliative therapy. Despite recent advance in medicine, survival rates in these patients are rarely higher than 5 years and prognosis remains poor. Our aim in this paper is to present the latest treatment options for oral squamous cell carcinoma emphasizing the monoclonal antibody therapy, oncolytic virotherapy and immunotherapy with IL2.
\end{abstract}

Keywords: oral squamous-cell carcinoma, monoclonal antibody therapy, oncolytic virotherapy, IL2

\section{Background}

In 2000, the American Cancer Society reported 30,200 cases of oral and pharyngeal cancer and 7,800 deaths in the United States. More than 30,000 new cases were recorded in $2003(2,3)$.

In Germany in 2009 More than 10,000 new OSCC patients were recorded, while 2012 saw 300,000 new cases worldwide (4).

According to a statistical survey conducted by the World Health Organization (WHO) in the United States, the number of fatal outcomes among both male and female patients between 55 and 74 years of age 
diagnosed with malignant growths of the lips, oral cavity, and pharynx was 3,900 in 2009 and 4,250 in 2010. The survey showed a considerable increase of oral cancer morbidity rates in Germany between 2009 and 2012. In Bulgaria, this disease accounts for 200 to 300 deaths each year (Figure 1) (5) .

Figure 1. No. of deaths - Malignant neoplasm of the lips, oral cavity and pharynx, both sexes, 55-74 years

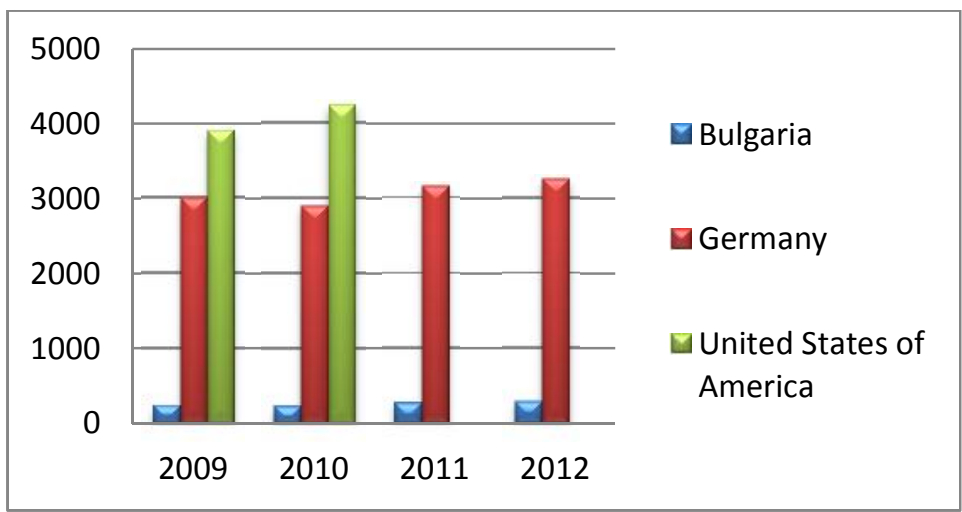

(WHO Mortality Database, March 2012)

Depending on the degree of differentiation of the cancerous cells, carcinomas are classified into well differentiated (G1), moderately differentiated (G2), poorly differentiated (G3) or non-differentiated (G4) (6)

OSCC prognosis depends on the grade and stage of the cancer and the patient's, sex and general condition. Unfortunately, survival rates in patients with oral cavity malignancies have not changed significantly over the past 30 years and prognosis remains poor despite advances in diagnosis and treatment approaches due to aggressive local invasions and metastasis, leading to recurrence. The 5-year survival rate for patients diagnosed with cancer of the oral mucosa is 55 percent. Depending on the tumour location, it varies from 86.5 percent for lip cancer to 48.1 percent for tongue cancer and 51.7 percent for other areas of the oral cavity (7).

A great deal of the cases relate to multiple cell anomalies resulting from chronic or excessive exposure to carcinogens associated most commonly with smoking, tobacco chewing, and alcohol consumption. We present the most frequently discussed predisposing causes for OSCC in Table 1 (8).

Table 1. Possible etiological factors for oral cancer $(2,3,8,9,10,11,12,13,14)$

\begin{tabular}{|l|}
\hline \multicolumn{1}{|c|}{ Predisposing causes } \\
tobacco \\
alcohol \\
HPV, EBV, HSV, CMV \\
genetic factors \\
diabetes \\
malnutrition \\
immune deficiency and candida \\
poor oral hygiene and mechanical \\
irritation and other factors \\
\hline
\end{tabular}




\section{Genetic factors \\ p53 and p16 as tumour suppressors}

In recent years, the causes underlying the development of squamous cell carcinoma are increasingly sought in patients' genetics. Modern research has not discarded long-known risk factors such as tobacco and alcohol but seeks the causes also in gene mutations and the presence of oncoviruses in patients with oral carcinoma.

OSCC arises as a consequence of multiple molecular events causing genetic damage affecting many chromosomes and genes, which leads to DNA changes. The accumulation of genetic changes leads to cell dysregulation to the extent that growth becomes autonomous and invasive mechanisms develop - this is carcinoma $(8,9)$. Genetic predisposition for SCC has been frequently discussed.

P53 is a protein involved in the control of cell proliferation and contributes to the maintenance of the cell's genetic stability. Under normal conditions, its level of concentration in the cell is very low and plays only a secondary role in the cell cycle. However, its accumulation has many effects:

- the mechanisms of DNA repair are activated;

- $\quad$ the cell cycle is stopped (15).

In the first case, following DNA damage, the proteins may disrupt the cell cycle at the end of the G1 phase and allow for DNA repair prior to replication. In malignant cells, the p53 coding gene has mutated and the mechanism described above does not function, which may result in insufficient DNA repair and subsequently to genetically unstable and potentially malignant cells. This explains why tumour cells are often more sensible to DNA-damaging agents such as radiotherapy or chemotherapy. This sensitivity may have a positive side effect after loss of p53 function which otherwise inhibits cell death $(15,16)$.

In the second case, since DNA repair becomes impossible, p53 induces cell apoptosis. P53 is a transcription factor for protein p21 production. In turn, p21 inhibits CDK4 and CDK2 necessary for the release of transcription factor E2F, which is essential for cell -cycle progression. In malignant cells, the protein acts as a tumour suppressor and can be used a tumour marker $(16,17)$. The possibility of using this protein to diagnose SCC is tempting though still unproven.

The protein $\mathrm{p} 16$ ( $\mathrm{p} 16 \mathrm{INK} 4 \mathrm{a}$ ) is also a tumour suppressor and plays a key role in regulating the cell cycle by slowing-down cell-cycle progression. The protein p16 inhibits cyclin-dependent kinases CDK4 and CDK6 thus preventing retinoblastoma protein $(\mathrm{Rb})$ phosphorylation (18).

$\mathrm{Rb}$ restricts the cell's ability to replicate DNA by preventing its progression from the $\mathrm{G} 1$ to the $\mathrm{S}$ phase of the cell division cycle thus acting as a tumour suppressor. Rb becomes inactive due to its phosphorylation by cyclin-dependent kinases CDK4 and CDK6 (18).

However, tissue samples from OSCC patients exhibit hypermethylation of p16 and Rb promoter regions. These changes result in the loss of the suppressor gene function, negative regulation of gene expression, and lower p16 levels. This process is believed to be responsible for the development of different forms of cancer (18).

\section{Oncogenic viruses}


Chronic viral infections of the mouth may also become an underlying cause of SCC. This hypothesis is backed by the fact that various amounts of virus genomes are frequently detected in tumour cells. Some viruses such as HPV (human papillomavirus) are carcinogenic in themselves, while others such as EBV (Epstein-Barr virus), HSV (herpes simplex virus) simply accelerate the progression of existing cancer (19).

Oral HPV infection has been identified as a risk factor in its own, since $65.5 \%$ of HPV DNA has been detected in SCC patients. HPV attacks the squamous cell epithelium by penetrating into the cells and inducing proliferative changes. Some virus types carry a low risk of progression to cancer (HPV-6, 11), while others present a higher risk (HPV-16, 18, 31, 45 etc.) (20). Tumor cells contaminated with oncogenic types of HPV exhibit excessive p16INK4a expression as a result of the Rb (gene) by oncoprotein E7. In clinical practice, the tumor's HPV status is often determined indirectly by immunochemical assessment of p16 overexpression. Nearly 100 percent of contaminated cancerous cells exhibit p16INK4a overexpression, while non-dysplastic epithelium does not express this biomarker (21).

HPV-related growths in the head and neck region are of key importance not only because of their clinical severity, but also because of their better prognosis compared to HPV-negative tumours. This is partly due to the higher sensitivity of HPV-related cancers to chemo- and radiotherapy (22).

EBV and HHV-6 have been found in 40 and 80 percent of OSCC cases, respectively (20).

Eglin and coworkes inverstigated RNA complementary to HSV and found it in 53\% of carcinoma biopsy specimens but in no biopsy specimens of normal oral mucosa from the same patients (23).

Jalouli et coworkers investigated the prevalence of human papillomaviruses (HPV), herpes simplex virus (HSV) and Epstein-Barr virus (EBV) in 155 OSCC from eight different countries from different ethnic groups, continents and with different socioeconomic backgrounds (24).

Of all the OSCCs examined, 55\% were positive for EBV, 35\% for HPV and 15\% for HSV. The highest prevalence of HPV was seen in Sudan (65\%), while HSV (55\%) and EBV $(80 \%)$ were most prevalent in the UK. In $34 \%(52 / 155)$ of all the samples examined, co-infection by two $(46 / 155=30 \%)$ or three $(6 / 155=4 \%)$ virus specimens was detected. The most frequent double infection was HPV with EBV in $21 \%$ $(32 / 155)$ of all OSCCs. The authors concluded that the significance of their findings must be put in relation to other risk factors and these observations warrant further studies to determine the possible role of viral infections and co-infections with HPV, EBV and HSV as risk markers for the development of OSCC (24).

\section{Oncotherapy in squamous cell carcinoma patients}

The earlierst the diagnosis of SCC, the better the outcome of the treatment and the greater the likelihood for the prognosis to be more favorable. The choice of treatment depends on tumour stage. 
Table 2. Staging for oral carcinoma

\begin{tabular}{|l|c|}
\hline Stage I & T1N0M0 \\
\hline Stage II & T2N0M0 \\
\hline Stage III & T3N0M0 \\
& T1-3N1M0 \\
\hline Stage IV & T4N0-3M0 M1 \\
\hline
\end{tabular}

UICC (Union for International Cancer Control)

\section{Surgical Basics}

Curative therapy is aimed at the complete cure of the disease. In the case of squamous cell carcinoma of the oral cavity it includes total resection of the tumour, regardless of its size. The greatest advantage of surgical resection is the possibility to perform specific and histological verification of resection material during surgery to reduce possible errors in treating tumours with unclear borders and makes it possible to remove the tumour completely thus significantly reducing the risk of recurrence. In advanced cases this treatment is often impossible to perform. Palliative therapy is then recommended $(8,9,13,14,25)$.

Palliative treatment is not aimed at curing the disease but rather at symptom relief and improving the patient's quality of life $(13,14)$.

$\mathrm{T} 1$ and T2 SCC are treated by R0 resection (corresponds to resection for cure or complete remission), i.e. complete tumour excision and removal of 1 to $2 \mathrm{~cm}$ of surrounding healthy tissue. Since squamous cell carcinoma presents a high risk of recurrence with a nearly 50 percent incidence, neck lymph dissection is recommended. In SCC with N0 ipsilateral selective supraomohyoid neck dissection (SOHND) Level I III is performed, while median line involvement (tumours of the tongue or mouth floor) require ipsilateral and contralateral SOHND Level I - III $(13,14,26)$.

SCC N+ is treated by ipsilateral modified radical neck dissection (MRND) Level I - V and contralateral SOHND Level I - III. Advanced SCC requires tumour resection and additional radical or modified ipsilateral and contralateral neck dissection Level I - V. Unfortunately, radical resection of surrounding healthy tissue is impossible in these tumours. Therefore adjuvant radio-, chemo- or radiochemotherapy is needed in these cases $(13,14,26)$. 
Table 3. Oncotherapy in squamous cell carcinoma $(13,14,26)$

\begin{tabular}{|c|c|}
\hline $\begin{aligned} 1, & 2 \\
\text { No } & \end{aligned}$ & $\begin{array}{l}\text { - R0 resection, i.e. complete tumor excision and removal of } 1 \text { to } 2 \mathrm{~cm} \text { of surrounding } \\
\text { healthy tissue } \\
\text { - ipsilateral selective supraomohyoid neck dissection (SOHND) Level I - III } \\
\text { - median line involvement (tumors of the tongue or mouth floor)- ipsilateral and } \\
\text { contralateral SOHND Level I - III }\end{array}$ \\
\hline $\mathbf{1}, 2$ & $\begin{array}{l}\text { - R0 resection } \\
\text { - ipsilateral modified radical neck dissection (MRND) Level I - V and contralateral } \\
\text { SOHND Level I - III. }\end{array}$ \\
\hline $\begin{array}{l}3,4 \\
\mathrm{~N}+\end{array}$ & $\begin{array}{l}\text { Combined therapy } \\
\text { 1. Version: } \\
\text { - Resection (R0, R1, R2 or en-bloc-Resektion) and radical or modified radical ipsilateral } \\
\text { and contralateral neck dissection Level I - V } \\
\text { - adjuvant radio-, chemo- or radiochemotherapy } \\
\text { 2. Version: } \\
\text { - Preoperative radio- or radiochemotherapy } \\
\text { - Resection, neck dissection } \\
\text { - Postoperative radio-, chemo- or radiochemotherapy }\end{array}$ \\
\hline
\end{tabular}

\section{Radiation therapy}

Malignancies are treated by high-energy rays including electromagnetic rays ( $\mathrm{X}$ and Gamma rays). Irradiation is commonly applied percutaneously (external beam radiotherapy or teletherapy). It is also possible to implant a radioactive material in the tumor area (brachytherapy) $(25,27)$.

Table 4. Types of radiation therapy

\begin{tabular}{|l|l|}
\hline Teletherapy & Radiation is applied outside the patient's body \\
\hline Brachytherapy & The radiation source is placed inside or directly on the body \\
\hline
\end{tabular}

Rays act on the cells' ability to divide and proliferate. Hence, tumour cells which present a higher rate of division are more strongly influenced than neighbouring tissues whose cells' ability to divide is lower. The faster the cells divide the more sensitive the cancer is to radiation.

Patients with stage III or IV oral SCC commonly receive adjuvant radiotherapy 6 to 8 weeks after tumor resection $(13,14)$.

The overall duration of therapy is 6 weeks according to the following schedule: $(13,14)$

- 2 Gy per session

- 5 sessions per week

- i.e. a total of 60 Gy

- patients who are at high risk of recurrence receive 70 Gy. 
Preoperative radio or radiochemotherapy is also an option. In this case, both resection and patient recovery are very difficult to achieve because of extensive tissue destruction and reduced blood flow. Therefore, this therapeutic approach often proves ineffective and is thus rarely used $(13,14)$.

\section{Chemotherapy}

Adjuvant chemotherapy for oral cancers can augment the survival benefit afforded by surgical management. The new chemotherapy medication in recent years is one of the major medical advances Most antitumor agents have expected and unexpected detrimental side-effects, usually affecting different structures in the maxillo-facial region (28).

Systemic chemotherapy is another treatment option for oral squamous cell carcinoma. Substances inhibiting cell division are commonly used. The decision about the type and form of treatment is made taking account of the tumor size and sensitivity, histological assessment, the presence of metastases, the patient's general condition and, last but not least, the patient's choice. Although squamous cell oral carcinoma is chemo-sensitive, it often fails to respond sufficiently. Therefore, chemotherapy is usually part of a combined treatment. Chemotherapy enhances the effect of radiotherapy, improves locoregional control, has a powerful effect on metastases and increases the patient's life expectancy $(13,14)$.

Commonly used drugs include 5-fluorouracil, vincristine, cisplatin, oxaliplatin, or carboplatin.

5-Fluorouracil belongs to the class of antimetabolite drugs. It is structurally similar to uracil, a pyrimidine base, and replaces it in RNA. In addition, 5- fluorouracil inhibits the thymidylat synthase, a key enzyme of biosynthesis. As a result, cell growth becomes inhibited, particularly for cells with a high rate of replication. Patients having received such therapy often present with mucositis $(13,14,26)$.

Cisplatin is a widely used cytotoxic agent which inhibits cell grow and proliferation. It is a member of the class of platinum complex-containing drugs which inhibit DNA replication by forming a cross-link between two neighbouring Guanine and/or Adenine bases in the DNA strands. It affects not only fast-growing tumor cells but, also, to a certain degree, healthy cells.

Vincristine (vinca-alkaloid) is a cytostatic drug used as an antimitotic agent in complementary or alternative treatment with other drugs for chemotherapy of mouth cancer. Vinca alkaloids bind to the protein tubulin and inhibit microtubule formation. Since microtubules have an important role in chromosome distribution in daughter cells during cell division, their absence results in cell death. Also, vinca alkaloids affect DNA and RNA synthesis. Vincristine induces p53 expression and apoptosis in several types of tumor cells (29).

Oxaliplatin and carboplatin are platinum-containing compounds with a wide-spectrum anticancer effect and potent preclinical and clinical activity in a large variety of tumours. They are also a possible choice in the treatment of OSCC, although they are not widely used (30).

\section{New technologies and combinations}

\section{Monoclonal antibody therapy}


The challenge in treating OSCC patients is to optimize treatment efficiency and simultaneously minimize treatment toxicity. A strategy to improve efficiency consists in adding monoclonal antibodies to classical chemotherapy (31). Monoclonal antibody therapy is a new approach in treating squamous cell carcinoma.

Cetuximab is a therapeutic monoclonal antibody of IgG type acting upon the epidermal growth factor receptor (EGFR). EGFR is a transmembrane receptor of epidermal growth factor with tyrosine-kinase activity, starting a cascade of signals, initiating signal transduction, regulating DNA synthesis, cell proliferation, adhesion, apoptosis, angiogenesis, cell migration, invasion, and metastasis. EGFR is found to overexpress in many tumours, including head and neck squamous cell carcinoma (HNSCC). The overexpression of EGFR is associated with poorer response to radiotherapy, increased tumor aggressiveness, faster disease progression, and low patient survival rates $(32,33)$. Tumor cells usually contain large amounts of EGFR in their cell membrane. Cetuximab binds to EGFR, thus inhibiting the receptor's activity and reducing the growth of tumor cells $(13,14,26)$. The clinical efficacy of cetuximab is based upon inhibiting the cell cycle progression, inducing apoptosis, inhibiting angiogenesis, inhibiting metastases, increasing the efficiency of chemotherapy, and radiotherapy, and inducing antibodydependent cell-mediated cytotoxicity. The drug has been approved for the treatment of SCC in 68 countries in combination with radio- and/or chemotherapy (31). The effect of cetuximab therapy is greater in patients with moderate immunoreactivity to EGFR compared to patients with EGFR overexpression. This may mean that patients with high EGFR overexpression should be treated with higher cetuximab doses (32).

Cetuximab was approved by the American Food and Drug Administration on 7 November 2011 in combination with radiotherapy for treating advanced NSCLC or in combination with cisplatin or carboplatin and 5-fluorouracil for primary treatment of patients presenting with recurrent or metastatic locoregional squamous cell carcinoma of the head and neck. In this case, the decision to approve cetuximab was based on the results of a large-scale international survey (EXTREME trial) involving 442 patients treated with cisplatin (100 mg/m2 intravenously) or carboplatin on day one and with 5-fluorouracil each 3 weeks (1000 mg/m2 intravenous infusion per day) and initial intravenous administration of cetuximab in a dose of $400 \mathrm{mg} / \mathrm{m} 2$, followed by weekly administration of $250 \mathrm{mg} / \mathrm{m} 2 \mathrm{i.v}$ for all patients. Overall survival rates and efficiency were considerably improved in these patients $(31,34)$.

Another survey conducted on 132 patients diagnosed with head and neck SCC present the combined cetuximab therapy and cisplatin. The patients having received combined cetuximab therapy of two 3week cycles $(400 \mathrm{mg} / \mathrm{m} 2$ intravenously on day one and then $250 \mathrm{mg} / \mathrm{m} 2 / \mathrm{wk})$ and cisplatin (75 or 100 $\mathrm{mg} / \mathrm{m} 2$ i.v. on day one each 3 weeks). Of these, 51 patients had stable disease (SD), 25 had progressing disease (PD/1), and 54 developed PD within 90 days after cisplatin therapy (PD/2). A high level of response to treatment was achieved in nine patients (18 percent) from the SD group, while in 39 (76 percent) disease control was less effective. From all PD/1 patients, five (20 percent) achieved partial remission and 11 (44 percent) were stabilized. Among PD/2 patients only three ( 6 percent) the tumour responded partially to treatment, and 25 (46 percent) had stable cancer $(32,33)$.

Cetuximab has been found to enhance the effect of cisplatin. Results have shown that including cetuximab in chemotherapy increased survival with 2 to 3 months compared with chemotherapy alone. However, cetuximab is not recommended in Scotland and England for this group of patients $(31,34)$. 
Interestingly, patients with p16-positive tumors treated with a combination of cetuximab and cisplatin exhibited higher survival rates than those with p16-negative tumors.

The side effects of cetuximab in combination with chemotherapy include nausea, anaemia, vomiting, neutropenia, rash, asthenia, diarrhoea, and anorexia. Conjunctivitis is observed in 10 percent of the patients on cetuximab. Other side effects are hypomagnesaemia, hypocalcaemia, and hypopotassaemia $(31,34)$.

\section{Oncolytic virotherapy}

Oncolytic viruses (OVs) are biotherapeutics that kill cells directly or indirectly after a viral infection. By modifying the viral genome, their toxicity can be directed toward tumour cells. They have been used experimentally in oncolytic virotherapy of cancer and have proven to be harmless for healthy cells. (35) OVs use cancer-related cell defects resulting from genetic disorders, including mutations and epigenetic reprogramming. These cell defects are responsible for a dysfunctional antiviral response and increase cell proliferation and metabolism thus creating a favourable environment for viral replication and OVs growth. In addition to the direct killing of cancer cells, OVs can also generate a strong anti-tumor immune response. Contaminated tumor cells induce the release of anti-inflammatory cytokines and stimulate the differentiation of antigen-presenting cells and T-cell activation (36).

A natural trend in the OV field has been to test OVs in combination with chemotherapeutics that are currently the standard of care. Classic chemotherapy drugs typically capitalize on the fact that cancer cells are continuously replicating unlike most normal cells. However, some normal cell types have higher replication rates, leading to significant off-target effects. Hematopoietic cells, among others, can be affected and this can lead to systemic immunosuppression (discussed below). While the evaluation of chemotherapeutic drugs in the context of OV therapy has been fairly empirical for the most part, their immunosuppressive effects can inherently complement OV activity by increasing OV spread within tumor beds and/or increasing anti-tumor immune responses (36).

A number of OVs are currently being evaluated in clinical trials to treat a range of cancer types. Of particular note, herpes simplex virus-1 (HSV-1), vaccinia virus, reovirus, and adenovirus-based OV strains have made the most progress toward approval. Shanghai Sunway Biotech oncolytic adenovirus (H101), deleted for the viral E1B gene and thought to target p53 deficient cancer cells, was the first approved OV in China as early as 2005, indicated for head and neck cancers (36).

The adenovirus was the first among oncoviruses to be approved by China's State Food and Drug Administration (SFDA) in 2005 for the treatment of head and neck cancers. Genetically modified $\mathrm{H} 101$ and Onyx-015 (dl1520) have been engineered to remove a viral defence mechanism that interacts with normal human gene p53. They do not specifically infect cancer cells, but they still kill cancer cells preferentially. There are data proving that the combination of $\mathrm{H} 101$ or Onyx-015 and chemotherapy is more efficient than chemotherapy alone. The effect is most conclusive when these viruses are directly injected into the tumor. Systemic therapy is recommended for metastatic disease (e.g. by intravenous infusion). This oncovirus is marketed under the brand name of Oncorine $(37,38,39,40)$.

HSV has a double-stranded DNA genome of $152 \mathrm{~kb}$ with three major gene regions: alpha, beta, and gamma. Each of these genes regulates the virus, i.e. its replication and proliferation in contaminated host 
cells. Oncolytic HSVs tested in the treatment of head and neck cancers include HSV HF10, HSV-171, and HSV- 1716. Injected tumors exhibit a higher level of infiltration with CD4-positive and CD8-positive cells compared to non-injected tumors. HSV-1716 was administered intratumorally ( $1 \times 105$ or $5 \times 105 \mathrm{PFU})$ to 20 SCC patients on day one, three, or fourteen prior to surgery. The treatment was well tolerated, without severe side effects and was associated with tumour necrosis. The two replications of the ICP34.5 gene (protein of herpes simplex virus type 1) are lacking; as a result, the gene is unable to replicate but its effect on cancer cells remains unchanged. Consequently, this seems to be an effective anti-tumor strategy $(35,41)$.

Successful use of OVs in anti-tumor therapy depends on the balance between the antiviral and anti-tumor immune response. The goal is to reduce the former in the short term and maximize the latter in the long term (36).

The combination of oncolytic virotherapy with chemotherapy, antibody therapy, immunotherapy, gene therapy, and/or radiation therapy has proven beneficial due to their synergistic anti-tumor effect. Combined therapies do not lead to cross-resistance. Chemo- and radiotherapy increase the expressions of some genes that are important for cell DNA repair which in turn increases HSV replication. Monoclonal antibodies enhance virus replication and stimulate apoptosis, thus becoming complementary to virotherapy $(35,42,43)$.

Oncolytic virotherapy using replication-competent viruses has attracted us as a new modality for cancer treatment. The fundamental concept of oncolytic virotherapy is that the viruses selectively replicate in and lyse tumor cells $(35,42,43,44)$.

Ogawa $\mathrm{F}$ and coworkers examined in vitro the effect of dual infection with herpes simplex virus type 1 (HSV-1) mutants on human oral squamous cell carcinoma. Human oral SCC cells were infected with gamma1 (34.5) gene-deficient HSV-1 R849 and HSV-1 HF that has multiple mutations and induces cell fusion. Oral SCC cells were infected with R849, followed by infection with R849 or HF. Virus production was elevated by both strains of HSV-1. Although the release of LDH from R849-infected cells was increased by secondary infection with R849 or HF, the effect of HF was more remarkable. When nude mouse tumors were treated with $\mathrm{R} 849$, $\mathrm{HF}, \mathrm{R} 849+\mathrm{R} 849$, or $\mathrm{R} 849+\mathrm{HF}$, treatment with $\mathrm{R} 849+\mathrm{HF}$ was the most effective. The conclusion of this study was that fusion-inducing virus HF enhances the oncolytic ability of gamma1(34.5) gene-deficient HSV-1 and provides a rationale for using fusogenic viruses as enhancing agents (43).

Katsura T et al. examined the effect of the histone diacetylase (HDAC) inhibitor trichostatin A (TSA) on the antitumor activity of a herpes simplex virus type-1 (HSV-1) mutant in oral squamous cell carcinoma (SCC) cells. Cytoplasmic nuclear factor-kappaB (NF-kappaB) component, p65, translocated into the nucleus after infection with $\gamma_{1} 34.5$ gene-deficient HSV-1 R849, indicating that R849 activated NFkappaB. TSA induced acetylation of p65 and increased the amount of p65 in the nucleus of oral SCC cells. Treatment of R849-infected cells with TSA also increased the amount of nuclear p65 and the binding of NF-kappaB to its DNA-binding site and an NF-kappaB inhibitor SN50 diminished the increase in nuclear p65. The viability of cells treated with a combination of R849 and TSA was lower than that of those treated with R849 only. After treatment with TSA, expression of the cell cycle kinase inhibitor p21 was upregulated and the cell cycle was arrested at G1. These results indicate that TSA enhanced the replication of the HSV-1 mutant through the activation of NF-kappaB and induced cell cycle arrest at G1 to 
inhibit cell growth. The conclustion of this study is that TSA can be used as an enhancing agent for oncolytic virotherapy for oral SCC with gamma 34.5 gene-deficient HSV-1 (42).

Nishiyama $Y$ and coworkers, have isolated a spontaneously occurring herpes simplex virus type 1 mutant, designated HF10, which efficiently replicates and induces cell fusion in most transformed cells, but is highly attenuated in mice. The authors found that HF10 can be used as an oncolytic virus for treatment of malignant tumors in various animal models. Clinical trials have shown that intratumoral injection of HF10 can induce extensive tumor cell death in patients with recurrent breast cancer and head and neck squamous cell carcinoma without significant adverse effects. The authors conclude HF10 is a promising agent for use in oncolytic virotherapy in non-central nervous system malignancies (44).

\section{Interleukin 2}

Interleukin 2 (IL-2) is a protein belonging to the class of cytokines. Its effect consists in stimulating and differentiating the T-cell response. Trial treatments for OSCC have been conducted with Interleukin 2 (IL2) administered at the tumor site or around tumor-draining lymph nodes eliciting an effective antitumor reaction. The mechanisms of nonspecific immunity first cause tumor shrinkage, whereas the onset of a tumor specific, delayed-type hypersensitivity and the onset of long-lasting immune memory are subsequent events. It inhibits the growth and division of cancer cells (45).

Local therapy: IL-2 therapy of cancer was originally introduced as a systemic therapy. This therapy led to about $20 \%$ objective responses. Systemic therapy however was very toxic due to the vascular leakage syndrome. Consequently a greater beneficial effect could be expected after local IL-2 application (peritumoral = juxtatumoral, intratumoral, intra-arterial, intracavitary, or intratracheal = inhalation). Local therapy with free IL-2 may induce rejection of very large, metastasized tumor loads, for instance advanced clinical tumors. The effects of even a single IL-2 application may be impressive. Not each tumor or tumor type is sensitive to local IL-2 application. For instance transplanted EL4 lymphoma or TLX9 lymphoma were not sensitive in our hands. Also the extent of sensitivity differs: in Bovine Ocular Squamous Cell Carcinoma often a complete regression is obtained, whereas with the Bovine Vulval Papilloma and Carcinoma Complex mainly stable disease is attained. Analysis of the results of local IL-2 therapy in 288 cases of cancer in human patients shows that there were $27 \%$ Complete Regressions (CR), $23 \%$ Partial Regressions (PR), 18\% Stable Disease (SD), and 32\% Progressive Disease (PD). In all tumors analyzed, local IL-2 therapy was more effective than systemic IL-2 treatment. Intratumoral IL-2 applications are more effective than peritumoral application or application at a distant site. If local IL-2 is combined with surgery, radiotherapy or local chemotherapy the therapeutic effect is usually greater than with either therapy alone. Hence local free IL-2 application can be recommended as an addition to standard treatment protocols. Local treatment with free IL-2 is straightforward and can readily be applied even during surgical interventions. Local IL-2 treatment is usually without serious side effects and besides minor complaints it is generally well supported. Only small quantities of IL-2 are required (46).

The changes in cytokine profile before and after local IL-2 administration. in cancer patients have been investigated. (47) After local injection, IL-2 probably leaks into the blood circulation. The higher increases of IL-2, IL-5 and IL-10 in ascites compared to the serum suggests that the injected cytokines and their effects are mainly local. The minor increase of the immunosuppressive IL-10 could explain the therapeutic difference between local and systemic IL-2 therapy since IL-10 levels markedly increase after systemic IL- 
2 therapy. IL-5 was always increased after IL-2 therapy and, consequently, may be a considered downstream mediator of antitumour responses (47).

Interleukin 2 (IL-2) administered at the tumor site or around tumor-draining lymph nodes elicits an effective antitumor reaction. The mechanisms of nonspecific immunity first cause tumor shrinkage, whereas the onset of a tumor specific, delayed-type hypersensitivity and the onset of long-lasting immune memory are subsequent events. It is mention that low doses of IL-2are effective, whereas no improvement or even a loss of efficacy follows the use of increasing doses (48).

Since 1975, Cortesina $G$ and coworkers have investigated the possibility of impairing the growth of squamous cell carcinomas (SCC) of the head and neck (SCCHN) by means of low doses of IL-2 injected around the cervical tumor-draining lymph nodes and not in the tumor area (49).

The Phase III trial was conducted to assess whether the disease free interval and overall survival of 202 patients with T2-T4,N0-N3,M0 squamous cell carcinoma of the oral cavity or oropharynx could be extended through the combination of surgery (and radiotherapy, if required) with perilymphatic recombinant IL-2 (rIL-2). The investigators injected five thousand units of rIL-2 around the ipsilateral cervical lymph node chain daily for 10 days before surgery. After surgery, contralateral 5-day rIL-2 courses were administered monthly for 1 year. The data emerging from this trial indicate that perilymphatic administration of low, nontoxic doses of rIL-2 is a simple and manageable way to delay recurrences of SCC (50).

The efficacy of ten daily injections of 500 or $500,000 \mathrm{U}$ of recombinant interleukin 2 (IL-2) given daily, at $1.5 \mathrm{~cm}$ from the insertion of the sternocleidomastoid muscle on the mastoid was evaluated in 31 patients with recurrent head and neck squamous cell carcinoma by $G$. Cortesinal and coworkers. No toxic effects were noted. One complete response (CR) and three partial responses (PRs) were observed in the 16 patients who received $500 \mathrm{U}$ of IL-2, whereas the higher dose was not effective. The duration of the responses was 3-5 months and additional courses of ten injections of IL-2 had no further effect (49).

A study was conducted on neoadjuvant treatment with IL-2 administered to 39 OSCC patients diagnosed with T2-3N0-2M0. Results have shown a general response to therapy in 42 percent of all subjects. Tumorinfiltrating CD4 + and reduction of CD8 + cells were observed. OSCC patients treated with IL-2 exhibited a marked change in tumor structure, infiltrating mononuclear cells, and increased CD4 +/ CD8 + ratio. The authors hypothesize that LI mode of action involves the combined activity of the different cytokines present in LI14 inducing a cascade of several events: tumor necrosis factors present in LI (such as tumor necrosis factor- alpha) attack the tumor to release tumor antigens; antigen-presenting cells (e.g., dendritic cells) transport the newly released tumor antigens to lymph nodes; lymphoproliferative cytokines (present in LI administered peri-tumorally and perilymphatically; eg, IL-1 and IL-2) induce a marked polyclonal expansion of tumor-specific T cells, primarily in lymph nodes; LI recruits CD4+ T cells from local lymph nodes via chemotactic factors and reverts the balance of intratumoral CD4+ and CD8+ cells in favor of CD4+ T cells, which further upregulates the antitumor immune response, resulting in tumor cell necrosis; LI recruits neutrophils (via GM-CSF, also present in LI), which propagates the destruction of the tumor cell nests; either Li-derived cytokines or the de novo cytokine production by the tumor-infiltrating cells induce massive local fibrosis. The investigators concluded that these immune-mediated processes continue long after the cessation of LI administration, as evidenced by the immune histopathologic changes observed 14 to 54 days after the end of $\mathrm{LI}$ treatment. The efficacy of this neoadjuvant treatment protocol for patients 
with advanced primary OSCC resulted in a marked reduction in tumor mass (in $42 \%$ of the patients) compared with baseline tumor measurements (45).

\section{Conclusion}

Although surgery, radiotherapy and chemotherapy haves advanced into the era of targeted drugs, the antitumor efficacy of current therapies is limited, most likely because of the high degree of cancer clonal heterogeneity, intratumoral genetic heterogeneity and cell signal complexity.

In the current review, we focus on advances in monoclonal antibody therapy, OV treatment and immunotherapy with IL2.

Successful contemporary combination therapy using monoclonal antibody therapy, OVs and IL2 with surgery, radiotherapy and chemotherapy will ultimately depend on effectively navigating the delicate balance between the anti-viral response and the anti-tumor immune response such as to minimize the former in the short term and maximize the latter in the long term.

\section{References}

1. Hu S, Arellano M, Boontheung $P$ et al. Salivary proteomics for oral cancer biomarker discovery. Clin Cancer Res 2008;14:6246-6252.

2. Sonis St, Fazio R, Fang L. Oral medicine secrets. Publisher: Hanley \& Belfus 2003

3. Little J, Falace D, Miller Cr et al. Dental Management of the Medically Compromised Patient, (6th ed), 2002, Mosby.

4. "Oral cancer statistics". Cancer Research UK. Retrieved 28 October 2014. http://www.cancerresearchuk.org/health-professional/cancer-statistics/statistics-by-cancertype/oral-cancer

5. http://apps.who.int/healthinfo/statistics/mortality/whodpms/)(http://apps.who.int/healthinfo/statistics /mortality/causeofdeath_query/)

6. Ugrinov R. Maxillofacial tumors in the cervical area. (In Bulgarian), 2009.

7. Listl S, Jansen L, Stenzinger A et al. Survival of Patients with Oral Cavity Cancer in Germany. PLoS ONE 2013; 8(1): e53415.

8. Scully C, Cawson R. Medical Problems in Dentistry.(4th ed)..Wright. 1998 book.

9. Scully C, Oral and Maxillofacial Medicine; The basis of diagnosis and treatment..Wright. 2004 book.

10. Cawson R, Odell E. Oral pathology and oral medicine, (8th ed), 2008.Churchill Livingstone. 
11. Cawson R, Binnie W, Barett A et al. Pathology of tumors of the oral tissues, Churchill Livingstone. 1998 book.

12. Mitchell D, Mitchell L. Oxford Handbook of Clinical Dentistry, (4th ed), Oxford. 2005 book.

13. Schwenzer N, Ehrenfeld M. Dental, Oral and Maxillofacial Surgery: Surgical Basics (In German), (4th ed). Thieme. 2008 book.

14. Schwenzer N, Ehrenfeld M. Dental, Oral and Maxillofacial Surgery: Oral and maxillofacial surgery (In German), (4th ed) Thieme. 2010 book.

15. Grundmann. Pathologie für Zahnmediziner. Urban \& Fischer. 2003 book.

16. Wernert $N$, Martini $M$, Berge $S$ et al. Molekularbiologische und immunhistochemische Untersuchung des tp53-Gens in menschlichen Ameloblastomen. mund- kiefer- und gesichtschirurgie. Mund-, Kiefer- und Gesichtschirurgie 2004; 8 (3):167-172.

17. Lin $\mathrm{Y}$, Clair J, Luo $\mathrm{J}$ et al. p53 modulates NF-kB mediated epithelial-to-mesenchymal transition in head and neck squamous cell carcinoma. Oral Oncol 2015;51(10):921-928.

18. Kohr G, Froemming G, Zain R. DNA Methylation Profiling Revealed Promoter Hypermethylationinduced Silencing of p16, DDAH2 and DUSP1 in Primary Oral Squamous Cell Carcinoma. Int J Med. 2013. doi:10.7150/ijms.6884: 1727-1739.

19. Krasteva A, Dobreva A, Galabov V et al. Human Papillomaviruses, Herpes Viruses and Oral Neoplasms, Medinform, 2015;1:131-141.

20. Krasteva , alvachev Z, iselova. Viral infections in the oral medicine (In Bulgarian), Medinform LTD, 2014 book.

21. Rikardsen $\mathrm{O}$, Bjerkli I, Uhlin $\mathrm{H}$ et al. Clinicopathological characteristics of oral squamous cell carcinoma in Northern Norway: a retrospective study 2014;14:103.

22. Marur S, D'Souza G, Westra W et al. HPV-associated head and neck cancer: a virus-related cancer epidemic The lancet oncology 2010;11:8: 781-789.

23. Eglin RP, Scully C, Lehner $\mathrm{T}$ et al. Detection of rna complementary to herpes simplex virus in human oral squamous cell carcinoma 1983;22: 8353: 766-768.

24. Jalouli J, Jalouli MM, Sapkota $D$ et al. Human papilloma virus, herpes simplex virus and epstein barr virus in oral squamous cell carcinoma from eight different countries. Anticancer Res 2012; 32:2:571-580.

25. Kruchten A. The squamous cell carcinoma of the oral cavity, a retrospective analysis of 215 cases with particular reference oncological results of multimodal therapy concepts and their side effects (In German), 2011. Giessen. 
26. German Cancer Society, the German Cancer Aid, Guideline: diagnosis and treatment of oral cavity cancer, (In German), 2012.

27. Weber T. Memorix Dentistry (In German), (3th ed). Publisher: Thieme 2010 book.

28. Takawa M, Masuda $\mathrm{K}$, Kunizaki $\mathrm{M}$ et al. Validation of the histone methyltransferase $\mathrm{EZH} 2$ as a therapeutic target for various types of human cancer and as a prognostic marker. Cancer Science $2011 ; 102: 7: 1298-1305$.

29. http://www.sigmaaldrich.com/catalog/product/SIGMA/V8879?lang=en\&region=BG

30. Espinosa M, Martinez M, Aguilar J. Oxaliplatin activity in head and neck cancer cell lines, Cancer Chemotherapy and Pharmacology.2005;55: (3): 301-305.

31. Psyrri A, Dafni U et al. Combining Cetuximab With Chemoradiotherapy in Locally Advanced Head and Neck Squamous Cell Carcinoma: Is More Better?, J Clin Oncol. 2014; 20:32: 29292931.

32. Herbst R, Arquette M, Shin D et al. Phase II Multicenter Study of the Epidermal Growth Factor Receptor Antibody Cetuximab and Cisplatin for Recurrent and Refractory Squamous Cell Carcinoma of the Head and Neck. J Clin Oncol. 2005;20:23:5578-5587.

33. Baselga J, Trigo J, Bourhis J et al. Phase II Multicenter Study of the Antiepidermal Growth Factor Receptor Monoclonal Antibody Cetuximab in Combination With Platinum-Based Chemotherapy in Patients With Platinum-Refractory Metastatic and/or Recurrent Squamous Cell Carcinoma of the Head and Neck. journal of clinical oncology 2005; 23:24:5568-5577.

34. Cohen M, Chen H, Shord St et al. Approval Summary: Cetuximab in Combination With Cisplatin or Carboplatin and 5-Fluorouracil for the First-Line Treatment of Patients With Recurrent Locoregional or Metastatic Squamous Cell Head and Neck Cancer. Oncologist. 2013;18:4:460466.

35. Shilpa P, Kaul R, Bhat S et al. Oncolytic Viruses in Head and Neck Cancer: A New Ray of Hope in the Management Protocol. Annals of Medical \& Health Sciences Research. 2014;4:9:178-184.

36. Forbes N, Krishnan R, Diallo J et al. Pharmacological Modulation of Anti-Tumor Immunity Induced by Oncolytic Viruses. Front Oncol 2014;4:191.

37. Heise C, Sampson A; Williams A et al. ONYX-015, an E1B gene-attenuated adenovirus, causes tumor-specific cytolysis and antitumoral efficacy that can be augmented by standard chemotherapeutic agents. Nature Medicine 1997;3: 39-645.

38. Kirn D. Oncolytic virotherapy for cancer with the adenovirus dl1520 (Onyx-015) results of phase I and II trials. Expert Opin Biol Ther 2001;1:3:525-38.

39. Frew E, Sammut $M$, Shore A et al. Chinese health biotech and the billion-patient market. Nature Biotechnology 2008;26 :1: 37-53. doi:10.1038/nbt0108-37. PMID 18183014. 
40. Garber, K. China Approves World's First Oncolytic Virus Therapy for Cancer Treatment. JNCI.2006; 98:5: 298-300. doi:10.1093/jnci/djj111. PMID 16507823.

41. Brown S, Dunn $P$, Singh $B$ et al. Methods for treating cancer using herpes simplex virus expressing antisense to the squamous cell carcinoma related oncogene. 2009

42. Katsura $T$, Iwai $S$, Ota $Y$ et al. The effects of trichostatin $A$ on the oncolytic ability of herpes simplex virus for oral squamous cell carcinoma cells. Cancer Gene Ther 2009;16:3:237-245. doi: 10.1038/cgt.2008.81.

43. Ogawa $\mathrm{F}$, Takaoka $\mathrm{H}$, Iwai $\mathrm{S}$ et al. Combined oncolytic virotherapy with herpes simplex virus for oral squamous cell carcinoma. Anticancer Res 2008;28: 6A:3637-3645.

44. Nishiyama Y, Goshima F. Oncolytic virotherapy using replication-competent herpes simplex viruses. Uirusu 2007;57:1:57-65.

45. Tímár , Ladányi A, Forster-Horváth C. Neoadjuvant Immunotherapy of Oral Squamous Cell Carcinoma Modulates Intratumoral CD4/CD8 Ratio and Tumor Microenvironment: A Multicenter Phase II Clinical Trial. Journal of Clinical Oncology 2005; 23:15:3421-3432.

46. Den Otter W, Jacobs JJ, Battermann JJ et al.. Local therapy of cancer with free IL-2. Cancer Immunol Immunother 2008;57:7: 931-950. doi: 10.1007/s00262-008-0455-z.

47. Tomova R, Pomakov J, Jacobs JJ et al. Changes in cytokine profile during local IL-2 therapy in cancer patients. Anticancer Res 2006;26:3A:2037-2047.

48. Forni G, Giovarelli M, Santoni A. Lymphokine-activated tumor inhibition in vivo. The local administration of interleukin-2 triggers non-reactive lymphocytes from tumor-bearing mice to inhibit tumor growth. J Immunol 1985;139:3933-4041.

49. Cortesina G, De Stefani A, Galeazzi E et al. Temporary regression of recurrent squamous cell carcinoma of the head and neck achieved with low but not with high dose of recombinant interleukin-2 injected perilymphatically. Br J Cancer 1994;69:572-576.

50. De Stefani A, Forni G, Ragona $R$ et al. Improved survival with perilymphatic interleukin 2 in patients with resectable squamous cell carcinoma of the oral cavity and oropharynx. Cancer 2002; 95:1:90 - 97.

\section{Corresponding author:}

Assya Krasteva

Faculty of dental medicine, Department of oral and imaging diagnostic, Medical University, Sofia, Bulgaria

1 Georgi Sofiyski blvd., 1431 Sofia, Bulgaria

e-mail:asyakrasteva@abv.bg 